\title{
Location Aided Broadcast in Wireless Ad Hoc Networks
}

\author{
Min-Te Sun, Wuchi Feng, Ten-Hwang Lai \\ Department of Computer and Information Science \\ The Ohio State University, Columbus, OH 43210, USA
}

\begin{abstract}
Building efficient ad hoc networks for wireless communications is challenging due to the dynamic nature of the hosts. Broadcast service in ad hoc networks is critical in supporting various applications and protocols. However, excessive redundant retransmissions of traditional broadcast protocols in mobile wireless ad hoc networks have caused the infamous "broadcast storm problem." Various broadcast protocols have been proposed to alleviate the broadcast storm problem. In this paper, we identify two primary design issues, namely defer time generation and redundant message classification, for all these protocols. We propose a Distance-based Defer Time scheme for the first issue and an Angle-based Scheme for the second issue. The two schemes together result in a broadcast protocol that enjoys flooding's high reachability and non-flooding schemes' bandwidth efficiency.
\end{abstract}

\section{INTRODUCTION}

As one type of wireless technology, ad hoc networks allow mobile hosts to route messages wirelessly without the aid of a fixed backbone network. Because a fixed wired network infrastructure is not required, the deployment of ad hoc networks can be fast and cheap. However, the lack of fixed infrastructure also means that the whole network topology can dynamically change as the mobile hosts move arbitrarily. Also, compared with wired networks, the bandwidth of wireless networks is scarce, making flooding-based protocols impractical. All these make message communications in ad hoc networks complicated and challenging.

There are different types of message communication services in ad hoc networks. Among them, the ability to broadcast message is a fundamental one. When a message is intended for all the participants of the ad hoc network (e.g., a warning message for all nodes) or when a message needs to reach a specific set of nodes quickly (unicast or multicast) but the route to the destinations is still unknown, a broadcast service is needed. Most of the advanced broadcast protocols, such as the tree-based protocol in [1], do not work well for ad hoc networks due to the dynamic nature of the network topology. The traditional broadcast implemented by flooding is used in a number of proposals [7] [8] [13] [15]. The major problem of flooding is the large bandwidth consumption caused by the excess number of retransmissions. To reduce the number of retransmissions, each message is assigned a timeto-live field (TTL) and each node maintains a list of previously received messages. Although the TTL and the received message list help prevent messages from staying within networks forever and eliminating duplicated retransmissions of the same message from a node, broadcast is still costly in terms of bandwidth consumption in ad hoc networks. Assuming the TTL is large and the number of nodes in the ad hoc network is $n$, the number of message retransmissions for a broadcast would be $n$ since all the nodes in the network will send the message exactly once when they receive it the first time. To make the situation worse, the retransmissions of close-by nodes for a broadcast are likely to happen at the same time due to the nature of broadcast. As a result, the retransmissions quickly lead to massive collisions and channel contention, causing more retransmissions be generated. This phenomenon is known as the broadcast storm problem in the literature [2].

In this paper, we address the broadcast storm problem. Reviewing existing solutions for the problem, we identify two major design issues: 1) defer time generation and 2) redundant message classification. Based on our analysis, we propose a distance-based scheme for defer time generation and an angle-based scheme for redundant message classification. The two schemes together result in a broadcast protocol that enjoys flooding's high reachability and non-flooding schemes' bandwidth efficiency. Simulation results confirm these strengths.

Our protocol, as well as some protocols in [2], rely on location information. There are several ways to obtain location information (e.g., using the Global Positioning System (GPS) or the network-based geolocation technique). As the price of GPS chip drops to few dollars, it is not unreasonable to assume that in the near future each node of an ad hoc network will be equipped with a GPS chip. When necessary, a node can inform other nodes of its location by encoding the location information (less than 20 bits) in outgoing messages.

\section{Existing Solutions to the Broadcast Storm PROBLEM}

To solve the broadcast storm problem, one would immediately come up with the following general approach: 
when a node $S$ receives a broadcast message $m$

if the message has been received before the message is dropped

else if TTL of the message has expired the message is dropped

else

node $S$ sets a defer time;

when the defer time expires, $S$ determines

whether or not to retransmit message $m$

This skeleton has been used in protocols proposed in [2] [3]. There are two design issues with the above approach.

1. How should $S$ set the defer time?

2. How should $S$ determine whether or not to retransmit message $m$ ?

The first issue seems relatively simple and, not surprisingly, a very simple strategy has been proposed: each node randomly selects a defer time [2] [3].

As for the second issue, it seems plausible to refrain a node from retransmitting a message if the transmission would be redundant. Along this line of reasoning, the question becomes how to characterize a redundant (re)transmission. Some schemes have been proposed:

- Random-based scheme [2]: A node randomly discards a retransmission (i.e., not to retransmit the message).

- Counter-based scheme [2]: A node discards a retransmission if, during the defer period, it overhears the same message from its neighbors for more than a prefixed number of times.

- Distance-based scheme [2]: A node discards its retransmission if it overhears a neighbor within a distance threshold retransmitting the same message during its defer period.

- Location-based scheme [2]: A node discards its retransmission if its position is inside the convex hull of the neighbors that transmitted the same message during its defer period.

- Cluster-based scheme [2]: If a node is not a cluster head, it simply drops the retransmission. Otherwise, the node uses any of the above scheme to determine if it will retransmit.

- Scalable Broadcast Algorithm [3]: A node maintains the local network topology within 2 hops. A node discards its retransmission if it finds all its neighbors have been covered by some of its neighbor's retransmissions during defer period.

\section{Motivation OF OUR Research}

Notice that the defer times generated at various nodes potentially determines the order in which retransmissions are made at various nodes. The strategy of randomly generating defer times, as proposed in [2] [3], would be plausible if the order of retransmissions is not important. The importance of the order of retransmissions in a large part depends on how a node determines whether or not to retransmit a message (i.e., the second issue mentioned in the preceding section). If, for instance, the random-based scheme is used to decide whether a retransmission is needed, then the order of retransmissions is not important. If, on the other hand, the location-based scheme is used, then the order of retransmission will play an important role in determining a redundant retransmission. We feel that the order of retransmissions is in general important, and the scheme used to determine the defer time will affect the performance of the resulting broadcast protocol. One goal of our research is to design a more effective strategy for setting the defer time.

In many broadcast applications, reachability is vitally important. Although flooding is considered bad in bandwidth consumption, it guarantees the highest reachability. When designing a broadcast protocol, it is desirable that the protocol be capable of achieving the same reachability as flooding.

The scalable broadcast algorithm [3] has potential to achieve high reachability. The algorithm, however, needs to discover and maintain the local network topology (within two hops) for every node. When the network topology changes frequently, the overhead will increase and may exceed the benefit of reduced retransmissions.

All the schemes presented in [2] - random-based, counterbased, distance-based, location-based, cluster-based - may occasionally drop a non-redundant retransmission. When a nonredundant retransmission is dropped in the first few hops, the effect may propagate to the following hops and the number of unreached nodes may amplify quickly hop after hop. It is not hard to see that none of these schemes can achieve the same level of reachability as flooding.

It is our goal to design a broadcast protocol that achieves the same level of reachability as flooding, while enjoying the same level of message efficiency as existing non-flooding broadcast protocols.

\section{Distance-based Defer Time}

Let us consider the issue of setting defer times. Nodes with a larger defer time are scheduled to retransmit a message later than those with a smaller defer time. Unless a node decides on redundant/non-redundant retransmission regardless of other nodes' retransmissions, the node with a larger defer time is more like to find its retransmission redundant than the node with a smaller defer time. Since the purpose of a retransmission is to forward the message to more nodes, it seems plausible to let a node covering more new area to retransmit the message earlier than the node covering less new area. Thus, 
instead of randomly choosing a defer time, we propose the following Distance-based Defer Time Scheme. When $S$ receives a broadcast message from $N$, it sets the defer time to a value inversely proportional to a power of $\|\overrightarrow{S N}\|$. That is,

$$
\text { defer time }=\text { Max_Defer_Time } \cdot\left(R^{\epsilon}-\|\overrightarrow{S N}\|^{\epsilon}\right) / R^{\epsilon}
$$

An appropriate value for $\epsilon$ is $\epsilon=2$. Assuming a uniform distribution of nodes over the area, the choice of $\epsilon=2$ will set various nodes' defer times uniformly over the interval [0, Max_Defer_Time].

\section{Angle-BASEd Scheme}

The second issue is how to identify a redundant retransmission. Since broadcast service requires high reachability, a retransmission should not be easily discarded unless the coverage area is known to be completely covered. To achieve the maximal reachability and fast computation, we propose the Angle-based Scheme to identify redundant retransmissions.

For a node $S$ and its neighbor $N$, we define the cover angle of $N$ to be the angle $\angle A S B$, where $A$ and $B$ are the intersections of the two circles centered at $S$ and $N$, respectively. Let $C$ be on $S$ 's circle such that $\overline{S C}$ is parallel to the x-axis ( $C$ is to the east of $S$ ). Let $\alpha$ be the degree of $\angle C S A$ (counterclockwise), and $\beta$ the degree of $\angle C S B$ (counter-clockwise). The interval $[\alpha, \beta]$ is referred to as the cover range of $N$. Figure 1 illustrates these definitions.

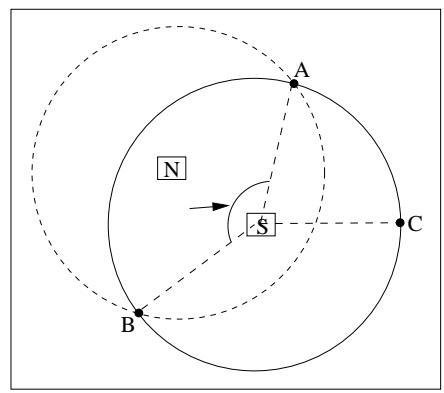

Fig. 1. Cover Angle

Assuming that all nodes have the same transmission radius, $R$, the values $\alpha$ and $\beta$ can be easily calculated as follows.

$$
\begin{gathered}
\angle C S N=\cos ^{-1}(\overrightarrow{S N} \cdot \overrightarrow{S C} /\|\overrightarrow{S N}\| \cdot\|\overrightarrow{S C}\|) \\
\angle A S N=\cos ^{-1}\left(\left(2 \cdot R^{2}-\|\overrightarrow{S N}\|^{2}\right) /(2 \cdot\|\overrightarrow{S N}\| \cdot R)\right. \\
\alpha=\angle C S N-\angle A S N \\
\beta=\angle C S N+\angle A S N
\end{gathered}
$$

If node $S$ overhears a retransmission from $N$, then $S$ knows that the sector from $\overrightarrow{S A}$ to $\overrightarrow{S B}$ has been covered by the retransmission of $N$ (therefore the name cover angle for $\angle A S B)$. Now, suppose that $S$ has heard the retransmission of a same message from a number of neighbors with cover ranges $\left[\alpha_{1}, \beta_{1}\right], \ldots,\left[\alpha_{k}, \beta_{k}\right]$. We claim that the transmission area of node $S$ has been completely covered by its neighbors' retransmissions if and only if the union of the neighbors' cover ranges is $[0,360]$; i.e., $\cup_{i}\left[\alpha_{i}, \beta_{i}\right]=[0,360]$. To see this, assume that $\cup_{i}\left[\alpha_{i}, \beta_{i}\right] \neq[0,360]$. Then there is a cover range $[\alpha, \beta]$ not in the union $\cup_{i}\left[\alpha_{i}, \beta_{i}\right]$. The area close to the border of the range $[\alpha, \beta]$ in the transmission area of $S$ must not be covered. Conversely, assume $\cup_{i}\left[\alpha_{i}, \beta_{i}\right]=[0,360]$. Since the distance between $S$ and its neighbor $N$ is less than or equal to the radius of $S$, the overlap between $S$ 's and $N$ 's transmission areas must contain the sector corresponding to the cover range of $N$ (i.e., the sector from $\overrightarrow{S A}$ to $\overrightarrow{S B}$ in Figure 1). Therefore, the whole transmission area of $S$ must have been covered by its neighbors' retransmissions.

From the above discussions, we obtain the following anglebased scheme: if during its defer period node $S$ receives the retransmissions of a same message from a number of neighbors with cover ranges $\left[\alpha_{1}, \beta_{1}\right], \ldots,\left[\alpha_{k}, \beta_{n}\right]$ and if $\cup_{i}\left[\alpha_{i}, \beta_{i}\right]=$ $[0,360]$, then $S$ will not further retransmit the message.

If all nodes have the same transmission radius, then each cover angle has at least $120^{\circ}$. Thus, no three cover angles (around $S$ ) are mutually disjoint. Because of this property, the union $\cup_{i}\left[\alpha_{i}, \beta_{i}\right]$ can be computed in $O(n)$ time, where $n$ is the number of cover ranges in the union. Thus, the angle-based scheme is more efficient in time complexity than the locationbased scheme of [2] which needs $O(n \log n)$ time to compute a convex hull.

One nice feature of the angle-based scheme is that it can be easily adopted to ad hoc networks where various transmission radii are possible. As long as a node encodes its transmission radius along with its location in the header of the message before transmission, a node can calculate the cover angle range according to the locations and transmission radii of the neighbors using the similar procedure. For instance, assuming the transmission radius of node $S$ and its neighbor $N$ are $R_{S}$ and $R_{N}$, respectively, the above formulas become:

$$
\begin{gathered}
\angle C S N=\cos ^{-1}\left(\overrightarrow{S N} \cdot \overrightarrow{S C} /\|\overrightarrow{S N}\| \cdot R_{S}\right) \\
\angle A S N=\cos ^{-1}\left(\left(R_{S}^{2}+R_{N}^{2}-\|\overrightarrow{S N}\|^{2}\right) /\left(2 \cdot\|\overrightarrow{S N}\| \cdot R_{S}\right)\right.
\end{gathered}
$$

The only exception is the case where one transmission area is completely contained in another transmission area. For instance, in Figure 2, the cover range of $S 2$ for $S 1$ should be 
$[0,0]$, whereas $S 1$ 's cover range for $S 2$ should be [0,360]. With this modification, the above "if and only if" statement will still hold valid.

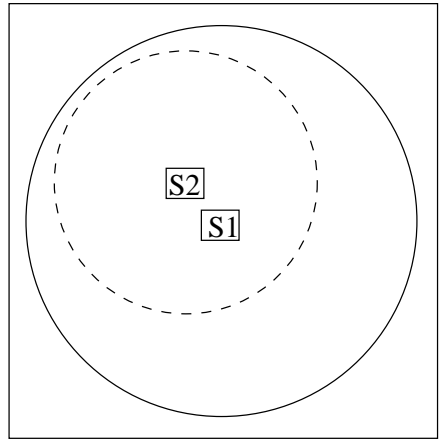

Fig. 2. Special Cover Angle Range

\section{Simulations AND ANAlyses}

To see how different defer-time-generation strategies and redundant-retransmission-classification schemes may affect the performance of broadcasting, we carried out a number of computer simulations. The following are the parameters used in the simulations:

Table 1: Simulation Parameters

\begin{tabular}{|c|c|}
\hline Items & Value \\
\hline Transmission Radius & 50 meters \\
Transmission Speed & $1000 \mathrm{bps}$ \\
Broadcast Message Size & $50 \mathrm{bits}$ \\
Defer Time Slot & $0.01 \mathrm{sec}$ \\
Neighbor Location & Uniform Distribution \\
\hline
\end{tabular}

We compared two defer time schemes: random defer time [2] and our distance-based defer time; and two redundant retransmission schemes: location-based scheme [2] and our angle-based scheme. Four different broadcast protocols based on different combinations of the above schemes were evaluated: 1) the location-based scheme with random defer time, 2) the location-based scheme with distance-based defer time, 3) the angle-based scheme with random defer time, and 4) the angle-based scheme with distance-based defer time. The first protocol is the one proposed in [2]. Two different time-to-live values, 2 hops and 5 hops, were used for simulations on different protocols.

A broadcast is said to have $100 \%$ reachability if it reaches all the nodes reachable by the flooding protocol. Since the angle-based scheme makes sure that all reachable area will be covered before dropping any retransmission, it always achieves a $100 \%$ reachability regardless of the defer-time scheme used. In contrast, since the location-based scheme does not cover all the area, it is suspected to often miss some nodes. Figures 3(a) and 3(b) show how often a broadcast protocol reaches $100 \%$ reachability. The $\mathrm{x}$-axis of the figures is the average number of neighbors for a transmission area, and the $y$-axis is the the number of broadcasts (out of 100) that reach $100 \%$ reachability. The figures indicate that the location-based scheme seldom achieves $100 \%$ reachability, especially for 5-hop broadcasts. For 2-hop broadcasts, the distance-based defer time scheme reaches $100 \%$ reachability more frequently than the random defer time scheme.
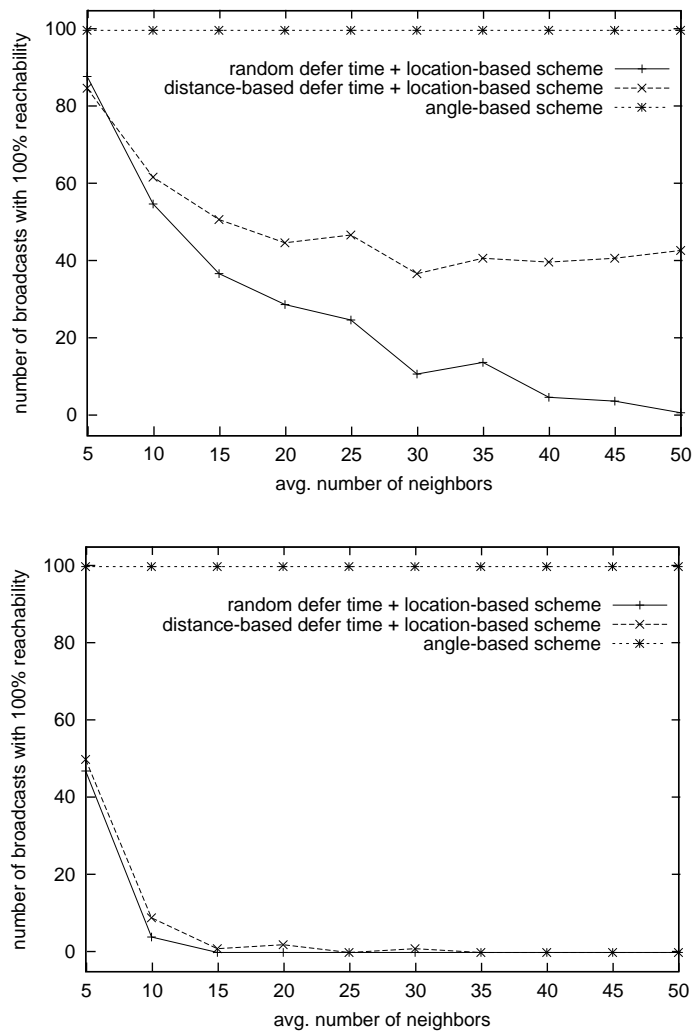

Fig. 3. Reachability: (a) 2-hop broadcast (b) 5-hop broadcast

Figures 4(a) and 4(b) show the average bandwidth consumption for 2-hop broadcasts and 5-hop broadcasts under different broadcast protocols. The $\mathrm{x}$-axis of the figures is the average number of neighbors for a transmission area, and the $\mathrm{y}$-axis is the average number of retransmissions for a broadcast The figures indicate that our distance-based defer time scheme reduces the number of retransmissions by $10-20 \%$ (as compared to the random defer time scheme). 
The angle-based scheme achieves $100 \%$ reachability by not dropping any non-redundant retransmissions. Therefore, it is expected to make more retransmissions than the locationbased scheme. Figures 4(a) and 4(b) show that our protocol, the angle-based scheme with distance-based defer time, generates much fewer retransmissions than flooding. The number of retransmissions for the protocol based on our schemes is comparable with that of location-based scheme protocol in [2], which does not provide reliable reachability.
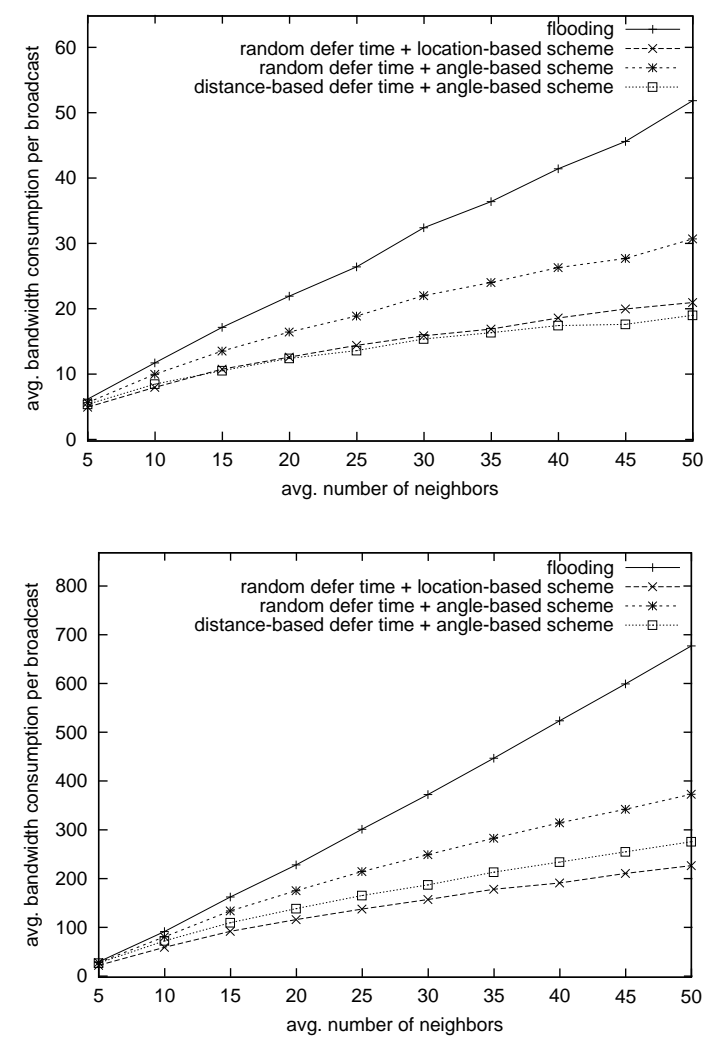

Fig. 4. Number of Retransmissions: (a) 2-hop broadcast (b) 5-hop broadcast

According to the simulation results, the distance-based defer time scheme improves both reachability and reduces the number of retransmissions. The angle-based scheme, although requires slightly more retransmissions than location-based scheme, constantly achieves $100 \%$ reachability. If the reachability is critical, our simulation results suggest the broadcast protocol using the angle-based scheme and distance-based defer time generation achieves $100 \%$ reachability with moderate bandwidth consumption.

\section{CONCLUSION}

We examined the broadcast storm problem and addressed the two important issues for solving this problem: defer time generation and redundant retransmission identification. We proposed a distance-based scheme for the first issue and an angle-based scheme for the second issue. The distance-based defer time creates a better order of retransmissions, resulting in a smaller number of retransmissions. The angle-based scheme is capable of achieving the same level of reachability as flooding. When the two schemes are used together, the resulting protocol enjoys flooding's high reachability and nonflooding schemes' bandwidth efficiency. The proposed protocol can be used even when nodes have different transmission ranges.

\section{REFERENCES}

[1] I. Chlamtac, S. Kutten, "Tree-based Broadcasting in Multihop Radio Networks," IEEE Trans. on Communications, Oct 1987.

[2] S. Y. Ni et al, "The Broadcast Storm Problem in a Mobile Ad Hoc Network," MOBICOM 1999.

[3] Wei Peng, Xi-Cheng Lu "On the Reduction of Broadcast Redundancy in Mobile Ad Hoc Networks," IEEE Proc. MobiHoc 2000

[4] A. Ephremies, T. Truong, "Scheduling broadcasts in multihop radio network," IEEE Trans. on Communications, Vol. 2, No. 4, Apr. 1990

[5] "http://www.ietf.org/html.charters/manet-charter.html"

[6] E. M. Royer, et al, "A Review of Current Routing Protocols for Ad Hoc Mobile Wireless Networks," IEEE Personal Communications, Apr. 1999

[7] C. E. Perkins, P. Bhagwat, "Highly Dynamic Destination-Sequenced Distance-Vector Routing (DSDV) for Mobile Computers," ACM SIGCOMM. Vol. 24, No. 4, Oct. 1994

[8] C. E. Perkins, "Ad Hoc on Demand Distance Vector (AODV) Routing," IETF, Internet Draft, draft-ietf-manet-aodv-00.txt, Nov. 1997

[9] S. Murthy, J. J. Garcia-Luna-Aceves, "An Efficient Routing Protocol for Wireless Networks," ACM Mobile Networks and Applications, Special issue on Routing in Mobile Communication Networks, 1996

[10] R. Ramanathan, M. Steenstrup, "Hierarchically-Organized, Multihop Mobile Wireless Networks for Quality-of-Service Support," ACM Mobile Networks and Applications, Vol. 3, No. 1, 1998

[11] Tsu-Wei Chen, Mario Gerla, "Scalable Routing Strategies for Ad Hoc Wireless Networks," IEEE JSAC, Aug. 1999

[12] B. Das et al, "Routing in Ad-Hoc Networks Using a Spine," IEEE Proc. ICCCN, Sep. 1997

[13] R. Sivakumar et al, "CEDAR: A Core-Extraction Distributed Ad Hoc Routing Algorithm," IEEE JSAC, Aug 1999

[14] Z. J. Haas, M. R. Pearlman, "The Performance of a New Routing Protocol for the Reconfigurable Wireless Networks," Proc. ICC, Jun. 1998

[15] Y. B. Ko and N. H. Vaidya, "Location-Aided Routing (LAR) in Mobile Ad Hoc Networks," MOBICOM 1998

[16] M. Sun, et al, "GPS-Based Message Broadcasting for Inter-vehicle Communications," Proc. IEEE ICPP 2000 